\title{
Producción científica internacional sobre gestión de proyectos en el área de Información y Documentación: 1996-2015
}

\author{
Silvia Cobo Serrano*
}

Artículo recibido:

7 de diciembre de 2015

Artículo aceptado:

29 de septiembre de 2016

\section{Resumen}

Se presentan los resultados de un estudio de producción sobre gestión de proyectos en el área de Información y Documentación, cuyo periodo cronológico abarca desde 1996 hasta el año 2015. La investigación sobre esta técnica de management se realiza a partir de los trabajos-fuente recuperados en la base de datos LISTA (Library, Information Science E Technology Abstracts). En esta primera aproximación a la temática, se analiza la evolución de la producción, la autoría de los trabajos-fuente (colaboración, productividad y género), la tipología documental e idioma de los mismos, la producción por publicación y la procedencia institucional y geográfica de los autores.

* Universidad Complutense de Madrid, España $\quad$ s.cobo@ucm.es

INVESTIGACIÓN BIBLIOTECOLÓGICA, vol. 32, núm. 75, abril/junio, 2018, México, ISSN: 2448-8321 pp. 125-144 
Palabras clave: Producción Científica; Análisis de la Investigación; Bibliometría; Gestión de Proyectos; Información y Documentación

\title{
International scientific production on projet management in Library and Information Science: 1996-2015 \\ Silvia Cobo-Serrano
}

\begin{abstract}
The paper aims to analyze the results of works published between 1996 and 2015 on project management in Library and Information Science. Analysis of this management technique examines information provided by the database LISTA (Library, Information Science \& Technology Abstracts). In this initial approach to the subject, the analysis focuses on the following indicators: production trends, authorship of the work-source (collaboration, productivity and gender), typology of documents, language, and production per publication, as well as institutional and geographical origin of authors.
\end{abstract}

Keywords: Scientific Production; Research Analysis; Bibliometrics; Project Management; Library and Information Science

\section{INTRODUCCIÓN}

L a industria de la información ha crecido de forma vertiginosa e, indiscutiblemente, en la segunda década del siglo XXI este cambio se ha hecho más evidente con el desarrollo de las tecnologías de la información, cuya incorporación a las unidades de información y documentación facilita la rápida adaptación a los nuevos cambios sociales y demandas de los usuarios.

De acuerdo con la literatura científica así como con la experiencia de los profesionales en las unidades de información y documentación, el medio adecuado para convertir estos cambios y demandas en realidad es la definición de la planificación estratégica (líneas estratégicas con proyección de futuro y objetivos generales), la planificación operativa (declaración de objetivos concretos) y, a su vez, la consecuente gestión de los proyectos definidos, 
que consiste en "la aplicación de conocimientos, habilidades, herramientas y técnicas a las actividades del proyecto para cumplir con los requisitos del mismo" (Projet Management Institute, 2008: 6).

Esta técnica del management ya fue expuesta por Corral (2000) cuando afirmó que la gestión de proyectos se configuraba como una competencia nuclear para la implementación de los planes y, en consecuencia, para alcanzar el cambio estratégico en las unidades de información y documentación. Por su parte, Moore (1998: 72-73) afirmó que la gestión de proyectos suponía la aplicación de procedimientos y herramientas tanto en las fases de planificación como en la etapa de seguimiento y control de los proyectos.

Otro aspecto importante es el conjunto de fases o etapas que facilitan la gestión de los proyectos. Con base en esta idea, MacLachlan (1996) manifestó la importancia que tienen el alcance, la planificación, la ejecución y el seguimiento de todos los proyectos. Además, Winston y Hoffman (2005: 5161) manifestaron la necesidad de aplicar principios de planificación, diseño, asignación de recursos y evaluación cuando las organizaciones se enfrentan a la gestión de un proyecto.

Asimismo, Carpenter (2011) enfatizó algunas de las etapas que forman parte del ciclo de vida de la gestión de los proyectos. La autora incluye en su definición las fases de inicio, planificación, organización y gestión del propio proyecto, el cual se basa en la puesta en común y en la utilización de un determinado conjunto de recursos para alcanzar los objetivos definidos dentro de la escala temporal acordada.

Con base en estos argumentos, se observa la importancia de dicha técnica en la formación de los profesionales de la información que tienen responsabilidades directivas en las unidades que lideran, pues son, mayoritariamente, los más proclives al uso de una metodología de trabajo basada en proyectos.

En consecuencia, la importancia de dicha técnica en la formación de los profesionales de la información y los constantes cambios sociales y demandas de los usuarios justifican la necesidad de estudiar la gestión de proyectos como medio para cumplir con la planificación estratégica de las unidades de información y documentación. Por ello, el presente trabajo tiene por objeto conocer el estado actual de la gestión de proyectos en el área de Información y Documentación a partir de un estudio de producción con el que se pretende conocer la evolución de la producción, la autoría de los trabajos-fuente (colaboración, productividad y género), la tipología documental e idioma de los mismos, la producción por publicación y la procedencia institucional y geográfica de los autores.

En la revisión de la literatura científica, se ha detectado la realización de un alto número de estudios de producción en el área de Información y 
Documentación que han permitido conocer la situación actual de una determinada disciplina, las autorías más productivas o la producción de una región geográfica. Por ello, se presenta a continuación una breve sistematización, con base en tres criterios (geográfico, temático e institucional), de los estudios de producción analizados, los cuales han servido para identificar los parámetros de análisis del presente trabajo de investigación.

En primer lugar y atendiendo a la región geográfica del estudio de producción, Jiménez-Contreras y Moya Anegón (1997: 252-266) analizaron a los autores científicos españoles en el área de Biblioteconomía y Documentación. Sus conclusiones pusieron de manifiesto que los organismos más productivos para el periodo analizado (1975-1995) eran el CSIC, las unidades informativas de las redes estatales y la universidad, siendo el CINDOC el organismo más productivo en términos proporcionales.

Por su parte y de forma individual, Jiménez-Contreras (2002) se encargó de estudiar la producción española en Información y Documentación difundida en revistas internacionales del Institute for Scientific Information (ISI). Como primer país productor destacó España en el contexto latinoamericano, alcanzando casi el 50 \% de la producción, y la séptima posición en Europa. Asimismo, resultó destacable Scientometrics como la publicación que absorbía "un tercio de todos los artículos publicados en este periodo" (Jiménez-Contreras, 2002: 6) y la universidad y el CSIC como procedencias académicas de la investigación española.

Posteriormente, Arquero Avilés y Salvador Oliván (2004: 53-74) también centraron su contribución en el contexto español con objeto de analizar la autoría de los trabajos publicados en revistas españolas para el periodo 1975 1984. Los resultados de la investigación pusieron de manifiesto el predominio de autores personales sobre los corporativos, el liderazgo de los autores españoles sobre los extranjeros y una falta de correspondencia entre el número de autores personales españoles y el número de trabajos publicados por cada uno de ello, entre otros aspectos. Asimismo, propusieron una clasificación de los autores españoles en cuatro grupos representativos (bibliométrico, universitario, bibliotecario y archivístico) y otra clasificación para las procedencias institucionales con tres niveles de especificidad. En términos generales, los autores concluyeron que el $40 \%$ de los trabajos estaban firmados por personal cualificado y que la Facultad de Ciencias de la Información de la Universidad Complutense de Madrid se erigía en representación del grupo universitario. Otra conclusión relevante aludía a que el $81 \%$ de los autores analizados habían escrito un único trabajo donde predominaba el corte profesional. 
En el contexto de América Latina y el Caribe, Licea de Arenas et al. (2000: 54-53) presentaron una visión del desarrollo de la investigación en Bibliotecología y Ciencia de la Información a partir de los resultados obtenidos en las bases de datos Library and Information Science Abstracts (LISA), Information Science Abstracts y Library Literature. Entre los resultados más relevantes se identificaron 1911 artículos únicos. Según el número de artículos indizados, destacaron geográficamente y de forma notable Brasil (53.6 \%), México (9.63\%), Cuba (6.64\%) y Colombia (5.19\%). Por su parte, 25 fueron los autores más productivos, quienes participaron en 234 artículos de los que el $25 \%$ fueron en coautoría. Por otro lado, las revistas en las que se publicaron la mayor parte de los artículos de la región fueron 13 (70 \% del total de artículos), siendo la autoría individual (76 \%) la modalidad preferida. Finalmente, los idiomas en los que publicaron los autores fueron el portugués (42\%) y el inglés ( $40 \%$ de los trabajos-fuente), respectivamente.

Moya Anegón y Herrero Solana (2002) se encargaron del análisis de la producción científica iberoamericana para conocer la visibilidad internacional de la misma. Para ello, utilizaron el Social Science Citation Index en el periodo 1991-2000, que se complementó con la base de datos Information Science Abstracts (ISA). Para abordar el estudio, los autores analizaron la producción por países, instituciones, citas por autor y citas por revista. Entre otras aportaciones, la Universidad Nacional Autónoma de México (UNAM) y el CSIC destacaron notablemente como instituciones productivas mientras que las tres grandes áreas temáticas de los trabajos consultados eran la bibliometría, las bibliotecas y la recuperación de información.

Por su parte, Herrero Solana y Ríos-Gómez (2006) analizaron la producción sobre Información y Documentación en Latinoamérica, utilizando la base de datos Social Science Citation Index en la categoría Information Science E Library Science para el periodo 1966-2003. Los autores concluyeron que la producción procedía principalmente de Brasil, seguido de México y Chile con gran diferencia. Asimismo, indicaron un grado bastante bajo de coautoría; señalaron la existencia de tres periodos delimitados con un crecimiento sostenido de la producción a partir de 1990; identificaron a la UNAM como la institución más productiva en Latinoamérica, Scientometrics como la publicación más destacada y a Macías Chapulas como el autor más productivo.

En 2008, Herrero Solana y Liberatore (2008: 230-239) analizaron el grado de visibilidad internacional de las revistas especializadas en Bibliotecología y Documentación en Iberoamérica. Del conjunto de revistas obtenidas (96), solamente el $37.5 \%$ fueron citadas en el ISI y el número total de 
citas halladas fue de 362. Por su parte, la Revista Española de Documentación Cientifica fue la publicación con mayor visibilidad (36.2\%), mientras que Brasil y España fueron los países mejor posicionados en el estudio.

Recientemente, la tesis doctoral de Liberatore (2015) ha tenido por objeto caracterizar el campo de la Bibliotecología y Ciencia de la Información en Brasil en el periodo 2000-2011. Para ello, el autor ha realizado un análisis empírico de la producción científica utilizando como fuente cuatro revistas brasileñas (Ciência da Informação, DataGramaZero: Revista de Ciência da Informação, Perspectivas em Ciência da Informação y Transinformação). Entre los resultados obtenidos se discuten indicadores relativos a la procedencia geográfica, institucional y disciplinar de los autores, productividad, coautoría, colaboración institucional así como otros indicadores de citación.

En 2006 también se publicó el artículo de Jiménez Contreras, Delgado López-Cózar y Ruiz Pérez sobre la producción española con visibilidad internacional en el Web of Science (WoS) entre 1995 y 2004. Los resultados pusieron de relieve la existencia de tres grupos de autores con respecto a la autocitación así como 171 revistas en las que se publicaron trabajos sobre Información y Documentación.

Finalmente, en este grupo de contribuciones se incluye el artículo de Ardanuy (2013: 1-7), quien se encargó de analizar la producción científica catalana, recogida en el Web of Knowledge, en Biblioteconomía y Documentación. De acuerdo con los resultados ofrecidos, el 88 \% eran artículos científicos; la temática predominante eran los estudios métricos, solamente un tercio de los autores eran mujeres, y se observó un alto predominio de la Universitat de Barcelona y un bajo volumen de producción en los trabajos catalanes en Información y Documentación.

Atendiendo al segundo criterio de clasificación, se pueden incluir en este grupo las contribuciones de Gómez Merino y Santaella Ruiz (2012) y la de Morena de Diago (2013). En el primer caso, los autores pusieron de manifiesto la escasa producción científica sobre estudios de usuarios en las investigaciones de Información y Documentación. Tras incluir algunas referencias históricas, analizaron 455 registros procedentes de la base de datos del ISOC-Biblioteconomía y Documentación del CSIC con objeto de conocer el peso de estos estudios en el área de conocimiento, los canales de comunicación, la productividad y cooperación entre los autores, las instituciones o el idioma más utilizado en la investigación sobre estudios de usuarios.

Morena de Diago (2013: 43-64) centró su estudio en la investigación cualitativa en el área de Información y Documentación en el periodo 1981-2010. Inicialmente, estableció el marco conceptual de la investigación cualitativa, proporcionó una contextualización del mismo en el área de conocimiento así 
como una breve revisión de la literatura científica sobre las técnicas cualitativas de recogida de datos. Además, realizó una propuesta de sistematización de las mismas en tres grandes grupos: técnicas conversacionales, técnicas documentales y técnicas observacionales. En línea con su estudio de producción, la autora identificó 521 trabajos-fuente procedentes de las bases de datos especializadas LISA (Library and Information Science Abstracts) y LISTA (Library, Information Science E Technology Abstracts), siendo clasificadas las fuentes de publicación más productivas en tres niveles de posicionamiento.

En tercer lugar y atendiendo al contexto institucional de la producción, Torres-Salinas, Delgado López-Cózar y Jiménez-Contreras (2009) analizaron la producción de las revistas de las facultades de Ciencias Sociales y Humanidades de la Universidad de Navarra (España) en el periodo 1999-2005. En su contribución, los autores abordaron el proceso de evaluación de la actividad científica de los profesores universitarios, comentaron los problemas existentes en estos procesos en el caso de los docentes en Humanidades y Ciencias Sociales y proporcionaron una breve explicación de los rankings IN-RECS (Índice de Impacto de las Revistas de Ciencias Sociales), IN-REJS (Índice de Impacto de las Revistas de Ciencias Jurídicas) y RESH (Índice de Impacto de las Revistas de Ciencias Humanas).

Por su parte, Berguillos y Fernández-Quijada (2012: 155) se encargaron de "analizar las dinámicas de producción científica en ciencias de la comunicación en las universidades de la Comunidad de Madrid entre 2007-2010”. En general, se puso de manifiesto que las universidades públicas eran más activas que las privadas así como el fuerte liderazgo de autoría de la Universidad Complutense de Madrid. Con respecto a las publicaciones, Icono 14, Estudios sobre el mensaje periodístico y Telos eran las revistas en las que se había publicado un mayor número de artículos firmados por autores adscritos a las universidades madrileñas.

Asimismo y según indicaron los autores, la coautoría mostraba una tendencia creciente, siendo la Universidad San Pablo CEU la que presentaba el índice más alto de este indicador. Tras presentar los tipos de autoría múltiple, la colaboración entre autores de la misma institución era la tendencia más habitual mientras que la Universidad Complutense de Madrid y la Universidad Rey Juan Carlos eran los centros de educación superior con más colaboraciones, liderando todas las tipologías la Universidad Complutense de Madrid, excepto la colaboración nacional, que compartía con la Universidad Rey Juan Carlos (Berguillos y Fernández-Quijada, 2012: 155-179). 
La investigación requirió de las siguientes fases y metodología de trabajo:

- Primera fase: delimitación conceptual de la materia y revisión de la literatura científica.

- Segunda fase: selección de la base de datos y definición de la estrategia de búsqueda. A la luz del uso de los estudios de producción en las investigaciones del área de Información y Documentación, se presentan los resultados de la presente investigación en la que la base de datos LISTA (Library, Information Science \& Technology Abstracts) fue seleccionada como fuente de información por reunir los siguientes requisitos: es una base de datos referencial, está especializada en Library and Information Science, tiene una amplia cobertura internacional, ofrece la mayor cobertura cronológica (desde el año 1960) e incluye el mayor número de registros indexados. Este recurso ya ofrecía, en el año 2006, "referencias procedentes de unas seiscientas publicaciones periódicas, actas de congresos y reseñas de monografías e informes de investigación" (Eíto, 2006: 437). Con respecto a la estrategia de búsqueda, el descriptor utilizado en los campos Término temático (Subject terms) y Resumen (Abstract or Author-supplied abstract) fue project management entrecomillado, y la cobertura temporal de los trabajos-fuente se estableció entre 1996 (fecha en la que aparecen los primeros manuales sobre gestión de proyectos en el área de Información y Documentación) y 2015.

- Tercera fase: análisis, eliminación de duplicidades y control de autoridades. Inicialmente, los resultados obtenidos fueron 250 registros. Tras la revisión y eliminación de las duplicidades identificadas, el estudio de producción se validó, finalmente, con 246 registros o trabajos-fuente. Asimismo, se realizó un proceso de control de autoridades y se tuvieron en consideración dos observaciones relativas a la procedecia institucional de los autores de los trabajos-fuente:

- Se optó por utilizar la institución más actual de aquellas autoridades que aparecían en varios registros con diferentes filiaciones institucionales.

- Se optó por consignar la primera filiación institucional en aquellos casos en los que aparecían varias en un mismo registro. 
- Cuarta fase: definición y análisis de los indicadores bibliométricos: evolución temporal de la producción, autoría de los trabajos-fuente, tipología documental, idioma, producción por publicación, procedencia institucional y procedencia geográfica.

- Quinta fase: discusión de resultados.

\section{RESULTADOS Y DisCUSIÓN}

\section{Evolución de la producción}

Se presenta la evolución de los trabajos sobre gestión de proyectos en Información y Documentación que son publicados en revistas, como resultado del análisis de los 246 trabajos-fuente recogidos.

De acuerdo con la cobertura temporal definida (1996-2015) y explicada en la segunda fase del apartado "Material y Métodos", la evolución de la producción se puede dividir en tres grandes periodos cronológicos (Figura 1): 1996-2003, 2004-2008 y 2009-2015.

El primer periodo se caracteriza por una escasa o nula producción que no supera el $5 \%$ del total de los trabajos-fuente. Por su parte, en la segunda etapa (2004-2008) se observa un notable ascenso de la producción que permanece de forma continuada hasta el 2008, año en el que se publican 28 trabajos sobre la materia objeto de interés. Concretamente, en este periodo tiene lugar la publicación de 98 contribuciones que suponen casi el $40 \%$ de la producción.

El tercer periodo, desde el 2009 hasta el año 2015, se caracteriza por un descenso en el volumen de publicación de trabajos. No obstante, los datos ponen de manifiesto el mantenimiento de la producción, concretamente entre 20 y 25 trabajos por anualidad. Específicamente, en este periodo se publican 132 trabajos, que representan en términos porcentuales el $53.66 \%$ de la producción total.

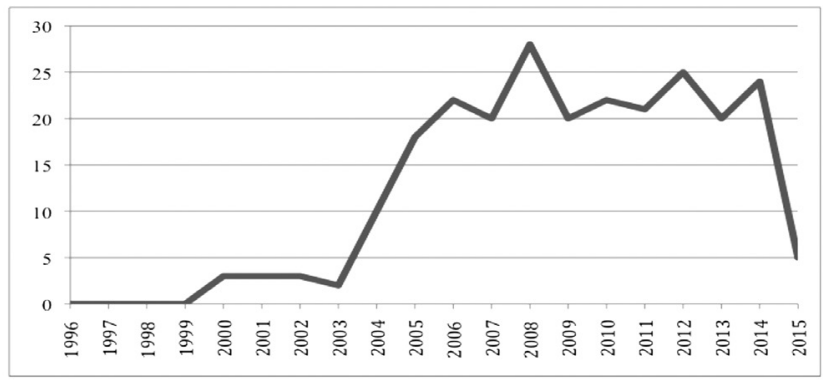

Figura 1. Evolución de la producción sobre gestión de proyectos en el área de Información y Documentación Fuente: elaboración propia 


\section{Autoría de los trabajos-fuente: colaboración, productividad y género}

Con el análisis de este indicador se pretende determinar, por un lado, el grado de colaboración de los autores en aquellos trabajos en los que la autoría es múltiple; por otro, la productividad de los mismos así como la proporción de autores de género masculino y femenino.

Atendiendo al primer parámetro de este indicador, se observa que la autoría individual de los trabajos-fuente supera a la autoría múltiple con un $55 \%$ de la producción analizada. En este sentido, la colaboración entre autores sigue sin ser la opción más habitual en la publicación científica, aunque cada vez es más frecuente la publicación de trabajos con más de una firma. En este caso, la autoría múltiple se ha localizado en el $44.13 \%$ de los trabajos-fuente, destacando el $22.52 \%$ de los mismos por ser firmados por dos autores. En total, se han cuantificado hasta nueve firmas por trabajo-fuente. En la Figura 2 se observa que la cobertura temporal se inicia en el año 2000 ya que entre 1996-1999 no se publicaron trabajos científicos sobre la materia, como muestra la Figura 1.

Con respecto a la evolución de la autoría (Figura 2), se observa una tendencia constante de trabajos-fuente firmados por autores individuales, siendo relevante en esta categoría de firmantes el año 2008 por la publicación de 15 contribuciones. En segunda y tercera posición, la Figura 2 pone de relieve el aumento de trabajos firmados por dos y tres autores. En el primer caso, el pico más alto se observa en los años 2010 y 2012 con siete trabajos. En el caso de tres firmas por trabajo, 2011, 2013 y 2014 son los años más productivos con cinco contribuciones por anualidad.

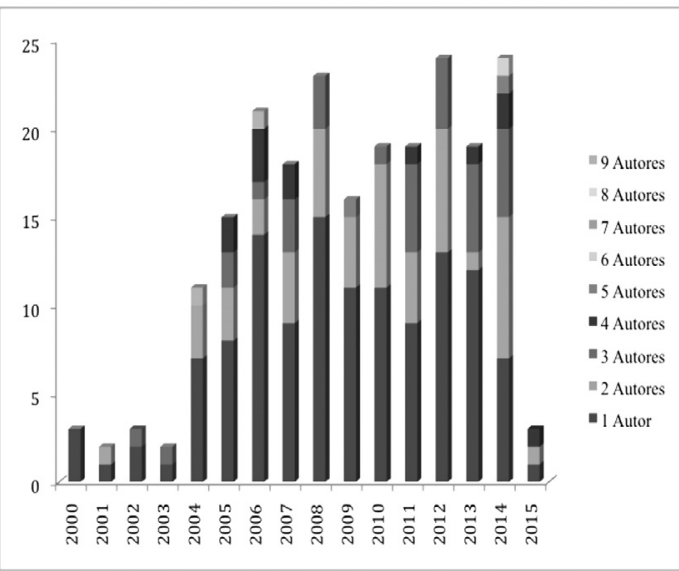

Figura 2. Evolución de la autoría de los trabajos-fuente Fuente: elaboración propia 
El segundo parámetro de interés en este indicador hace referencia a la productividad de los autores. Los resultados ponen de manifiesto que el $96.48 \%$ de los autores únicamente han publicado un trabajo sobre gestión de proyectos en el área de Información y Documentación entre 1996 y 2015. Por otro lado, un único firmante (H. Frank Cervone) ha sido el autor de 17 trabajos, cuyas publicaciones abarcan diversos aspectos: gestión de proyectos en bibliotecas digitales, metodologías estándar, desarrollo de casos de negocio, comunicación efectiva, habilidades para la gestión del cambio y del estrés, uso del análisis costo-beneficio, gestión del riesgo o metodologías ágiles en la gestión de proyectos.

Finalmente, se puede comentar que el $51 \%$ de los 369 autores identificados son de género masculino mientras que el $43 \%$ son de género femenino. Para su identificación se ha procedido a la búsqueda de los mismos en redes sociales profesionales, páginas web personales y páginas web institucionales. No obstante, se debe señalar que en 24 casos no ha sido posible la identificación del género de los autores.

\section{Tipología documental e idioma}

El objetivo de este indicador es doble: por un lado, se pretenden conocer los diferentes tipos de documentos en los que se publican los trabajos-fuente sobre gestión de proyectos enmarcados en el área de Información y Documentación; por otro lado, el análisis del indicador permitirá detectar la tipología documental más utilizada o predominante para la publicación de contribuciones sobre la temática.

El análisis de los trabajos-fuente ha concluido con la identificación de siete tipos de documentos (abstract, article, book review, calendar, case study, proceeding y product review), siendo indiscutiblemente reseñable el artículo como tipología dominante de la producción al superar el 80 \% de la misma. Seguidamente, aunque con bastante diferencia en lo que a número de trabajos-fuente se refiere, se encuentra la reseña de libros como segunda tipología destacable con 26 contribuciones (aproximadamente, $11 \%$ ). El resto de tipologías tiene porcentajes poco significativos (1\%-3\%).

Con respecto al idioma de los trabajos-fuente, este análisis facilita la identificación del conjunto de lenguas y detecta aquella más representativa o utilizada para la publicación de contribuciones científicas sobre gestión de proyectos en el contexto de los estudios de Información y Documentación.

Para este análisis, se estimó la proporción de contribuciones en lengua inglesa frente al resto de contribuciones en otros idiomas, dado que 231 trabajos-fuente estaban escritos en inglés, lo que derivó en que el $6 \%$ restante de la producción estuviese escrito en ocho idiomas distintos: alemán, español, 
chino, checo, francés, húngaro, persa y portugués, siendo el más representativo del conjunto el alemán y el español al representar más del $1.5 \%$.

\section{Producción por publicación}

Resulta de gran interés conocer las revistas en las que tiene lugar la publicación de los trabajos-fuente analizados así como aquellas que acogen la mayor producción de los mismos.

Tras la identificación de 120 revistas académicas, de las que casi el $60 \%$ sólo han publicado un único trabajo, resulta destacable el conjunto de 32 revistas que han publicado dos trabajos-fuente $(26.67 \%$ ) así como las 19 restantes, que son las que más trabajos publican (Tabla 1). En relación a estas últimas y de forma detallada, las revistas se disponen en función del número de trabajos-fuente publicados.

\begin{tabular}{|c|c|}
\hline Trabajos-fuente & Revistas \\
\hline 20 & OCLC Systems \& Services \\
\hline 12 & Information Systems Management \\
\hline 12 & Proceedings of the European Conference on Knowledge \\
& Management \\
\hline 10 & Sci-Tech News \\
\hline 8 & Journal of Information Systems Education \\
\hline 6 & AllM E-DOC \\
\hline 6 & Choice: Current Reviews for Academic Libraries \\
\hline 4 & Information Management \& Computer Security \\
\hline 4 & Records Management Society Bulletin \\
\hline 4 & Archivar \\
\hline 3 & Catalogue \& Index \\
\hline 3 & College \& Research Libraries \\
\hline 3 & Information Today \\
\hline 3 & International Journal of Information Management \\
\hline 3 & Joumal of Information Technology \\
\hline 3 & Journal of Library Administration \\
\hline 3 & Library Hi Tech \\
\hline 3 &
\end{tabular}

Tabla 1. Producción por publicación de los trabajos-fuente 
En virtud de las revistas identificadas, resulta interesante conocer las publicaciones del estudio que tienen impacto entre la comunidad académica. Para ello, se ha utilizado el Journal Citation Report (JCR), herramienta utilizada para conocer los indicios de calidad que está incluida en el Web of Science. Tras filtrar por la categoría Information Science and Library Science y utilizar la edición Social Science Citation Index del año 2014, se ha obtenido un conjunto de 84 revistas académicas con factor de impacto. Tras su comparación con las 120 publicaciones del estudio de producción, resultan coincidentes las siguientes 15 revistas cuyo orden de presentación responde a su posición en el JCR en relación al factor de impacto que tienen: Journal of Information Technology, Journal of the American Medical Informatics Association, Government Information Quarterly, International Journal of Information Management, Information Processing \& Management, College \& Research Libraries, Journal of Documentation, Aslib Proceedings, Library Hi Tech, Library Resources E Technical Services, Journal of Academic Librarianship, Information Research, Reference \& User Services Quarterly, Libri y Econtent.

\section{Procedencia institucional de los autores de los trabajos-fuente}

Para conocer el conjunto de instituciones a las que pertenecen los autores de los trabajos-fuente, se han analizado las filiaciones institucionales mencionadas en las contribuciones (en total 182 instituciones), que han sido clasificadas en función del número de trabajos-fuente en los que aparecían.

De acuerdo con la información consignada en la Tabla 2, existen cuatro categorías de instituciones: las que aparecen en un único trabajo (91.76 \% de las instituciones), las que aparecen en dos trabajos (6.6 \% de las instituciones), las que aparecen en tres trabajos $(1.1 \%)$ y, por último, una institución que figura en 17 trabajos-fuente $(0.55 \%)$, por lo que resulta la más relevante del conjunto.

\begin{tabular}{|c|c|c|}
\hline Trabajos-fuente & Instituciones & $\%$ \\
\hline 1 & 167 & 91.76 \\
\hline 2 & 12 & 6.6 \\
\hline 3 & 2 & 1.1 \\
\hline 17 & 1 & 0.55 \\
\hline Total & 182 & 100 \\
\hline
\end{tabular}

Tabla 2. Relación entre las instituciones de los autores y los trabajos-fuente 
Centrando la atención en las 15 instituciones con mayor frecuencia de aparición (estas son, según la Tabla 2, aquellas que figuran en 2, 3 y 17 trabajos-fuente), resulta de interés saber cuáles son, su tipología y procedencia geográfica, lo cual se detalla en la Tabla 3.

\begin{tabular}{|c|c|c|c|}
\hline Trabajos-fuente & Institución & Tipo de institución & Ubicación geográfica \\
\hline 17 & Cervone and Associates & Empresas y entidades & Estados Unidos \\
\hline 3 & $\begin{array}{l}\text { National Technical } \\
\text { University of Athens }\end{array}$ & Universidad & Grecia \\
\hline 3 & University of Houston & Universidad & Estados Unidos \\
\hline 2 & $\begin{array}{c}\text { Florida International } \\
\text { University }\end{array}$ & Universidad & Estados Unidos \\
\hline 2 & SINTEF & Empresas y entidades & Noruega \\
\hline 2 & $\begin{array}{c}\text { Australian National } \\
\text { University }\end{array}$ & Universidad & Australia \\
\hline 2 & McMillan LLP & Empresas y entidades & Canadá \\
\hline 2 & $\begin{array}{l}\text { University of Illinois } \\
\text { at Urbana-Champaign }\end{array}$ & Universidad & Estados Unidos \\
\hline 2 & University of Washington & Universidad & Estados Unidos \\
\hline 2 & Shahid Sattari Air University & Universidad & Irán \\
\hline 2 & University College Dublin & Universidad & Irlanda \\
\hline 2 & Intranet Focus Ltd & Empresas y entidades & Reino Unido \\
\hline 2 & University of Kent & Universidad & Reino Unido \\
\hline 2 & University of Reading & Universidad & Reino Unido \\
\hline 2 & University of Pretoria & Universidad & Sudáfrica \\
\hline
\end{tabular}

Tabla 3. Identificación, tipología y procedencia geográfica de las 15 instituciones con mayor frecuencia de aparición

Fuente: elaboración propia

En términos generales, se puede decir que la mayoría de los autores trabajan en universidades o empresas ubicadas en Europa y América, concretamente en Reino Unido y Estados Unidos.

Si se traslada el análisis del tipo de institución a la totalidad de instituciones (182), éstas se han clasificado en cinco grandes grupos: Universidades, Otros centros de enseñanza, Unidades de Información y Documentación (UID), Empresas y entidades y, por último, Organismos del sector público. Se ha incluido la categoría Otros para consignar aquellas instituciones que no tienen cabida en las categorías anteriores. 
Analizadas las instituciones de los trabajos-fuente, el $70 \%$ son universidades frente al $30 \%$ restante, que se mantiene en porcentajes similares sin superar el $10 \%$ (Figura 3).

El $43 \%$ de las universidades son estadounidenses y el $12 \%$ proceden de Reino Unido. Seguidamente, son representativas las universidades chinas, canadienses, alemanas y australianas con el $5.55 \%$ y el $4.76 \%$ en los dos últimos casos, respectivamente.

En segundo lugar, se han de mencionar las empresas y entidades que representan el $10 \%$ del conjunto, entre las que destacan Adhere Solutions, Inc. (Estados Unidos); Eli Lilly and Company (Estados Unidos); High Performance Technologies Inc. (Estados Unidos); Project Management Solutions, Inc. (Estados Unidos); Robbins-Gioia, LLC (Estados Unidos); Six Sigma Associates, LLC (Estados Unidos); William Blair \& Co., (Estados Unidos); Butler Group (Irlanda); CLS Communication AG (Suiza); McMillan LLP (Canadá); Intranet Focus Ltd (Reino Unido) y Cervone and Associates (Estados Unidos).



Figura 3. Tipo de institución en la que trabajan los autores Fuente: elaboración propia

En tercera posición, se sitúan otros centros de enseñanza con el $9 \%$ del conjunto de las instituciones. En este caso, forman parte del grupo EBS Business School (Alemania), Frankfurt School of Finance \& Management (Alemania), Fundação Getulio Vargas - Escola de Administração de Empresas 
de São Paulo (Brasil), Mohawk College (Canadá), Institute of Scientific and Technical Information of China (China), Centro de Investigaciones y Servicios Ambientales y Tecnológicos (Cuba), Instituto de Información Científico Tecnológica (Cuba), Babson College's Center for Information Studies (Estados Unidos), ESC Toulouse Business School (Francia) o el Royal Institute of Technology (Suecia).

Por último, otro conjunto de instituciones relevantes son las incluidas en el grupo de clasificación relativo a las unidades de información y documentación. Aunque representan el $4 \%$ del conjunto de instituciones, se pueden citar Calgary Public Library (Canadá), Jacksonville Public Library (Estados Unidos), Library of Congress (Estados Unidos), Queens Library (Estados Unidos), Bibliothèque nationale de France (Francia) y la Wellcome Library (Reino Unido).

\section{Procedencia geográfica}

Se trata del último indicador del estudio de producción con objeto de identificar los países en los que trabajan los autores de los trabajos-fuente. Para ello, se ha procedido a la ubicación geográfica de las filiaciones instituciones reflejadas en sus respectivas contribuciones.

De acuerdo con la Figura 4, se han identificado 30 países, de los que aproximadamente el 50 \% están ubicados en Europa, el $30 \%$ en Asia, el $14 \%$ en América y el $3 \%$ en África. De todos ellos, destaca significativamente Estados Unidos, país en el que se localizan 74 instituciones de carácter universitario y comercial. Por su parte, el segundo país en el que se concentran mayor número de instituciones es Reino Unido con 24 y, finalmente, en tercera y cuarta posición se encuentran Canadá y Alemania con 11 y 9 instituciones, respectivamente. En consecuencia, en posiciones inferiores se encuentran los 26 países restantes cuyo número de instituciones oscila entre 1 y 8 .

\section{Conclusiones}

Del estudio realizado, se observa escasa y tardía producción de obras que versan exclusivamente sobre gestión de proyectos en Información y Documentación, a pesar de su importancia manifiesta en la profesión. En términos bibliométricos, el volumen total de trabajos publicados, en torno a 250, es bajo, a pesar de la amplia cobertura cronológica analizada. En este sentido, las primeras publicaciones monográficas datan de 1996 mientras que el pico de producción más alto tuvo lugar en 2008. 


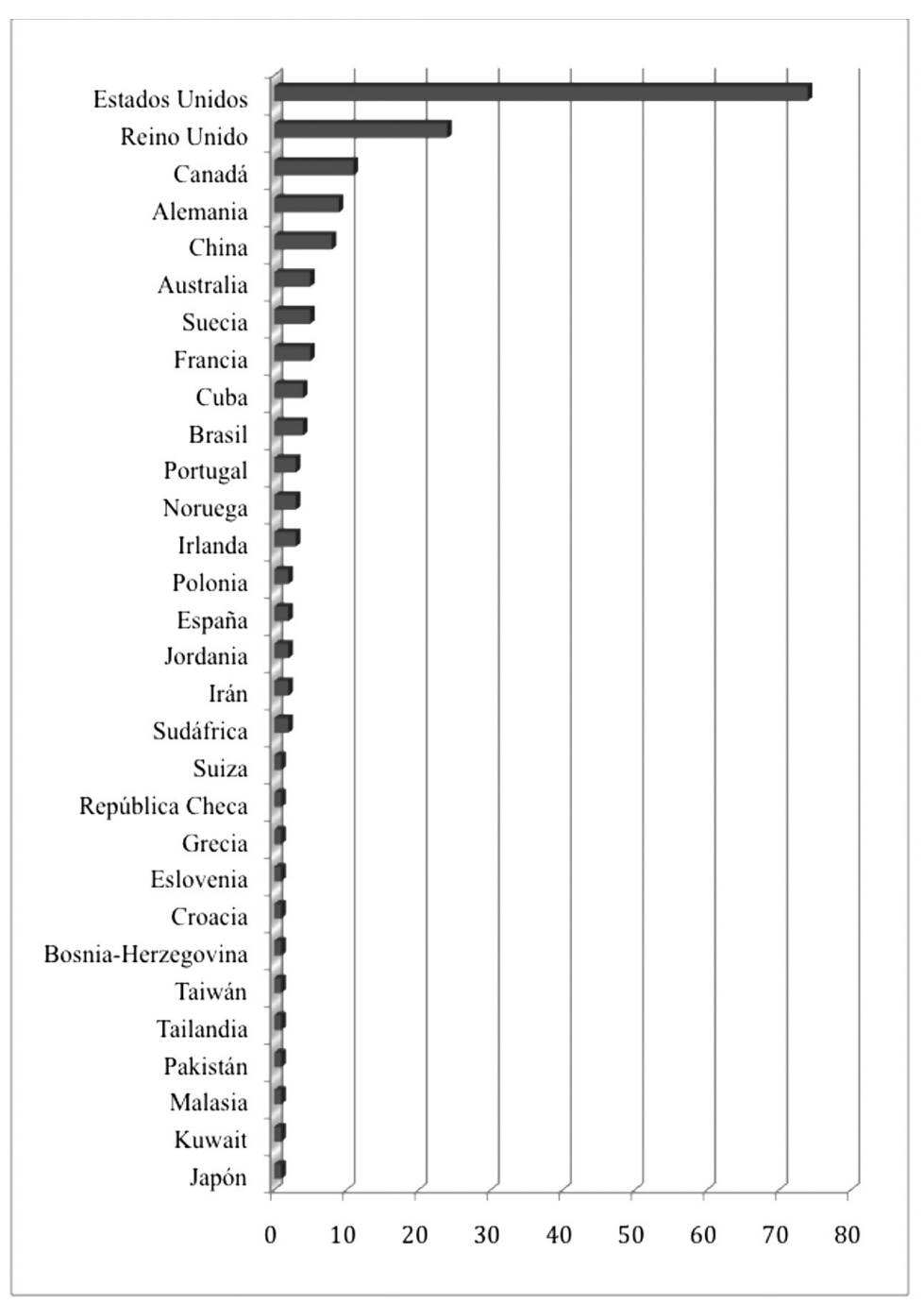

Figura 4. Procedencia geográfica de las instituciones de los autores de los trabajos-fuente Fuente: elaboración propia

Asimismo, la evolución de la producción de trabajos que versan sobre gestión de proyectos en el área de Información y Documentación indica que se trata de una temática consolidada y en expansión.

La materia, por sí misma, propicia que la autoría de los trabajos publicados sea múltiple, dado el carácter colaborativo de la gestión de proyectos, pues los resultados obtenidos indican que, aproximadamente, el $45 \%$ de los 
trabajos-fuente analizados fueron firmados por dos o más autores. En virtud de esta situación, sería muy recomendable que los directores o gestores de proyectos de las unidades de información y documentación publicaran la metodología o proceso de gestión de aquellos proyectos que implementan en dichas unidades.

La publicación de los trabajos que versan sobre gestión de proyectos en Información y Documentación tiende a ser inmediata. Por ello, el $82.93 \%$ de las contribuciones son artículos, mayoritariamente en inglés, publicados en revistas académicas (concretamente, 15 están incluidas en el Journal Citation Report), que son el canal de difusión más utilizado por los autores, quienes proceden indiscutiblemente del ámbito universitario.

Ante la falta de trabajos publicados sobre gestión de proyectos en lengua española así como firmados por autores españoles en el área de Información y Documentación, se impone la necesidad de fomentar la investigación y posterior publicación de trabajos que versen sobre la temática, ya que tiene una importancia nuclear para la adecuada formación de los profesionales de la información, que con mayor frecuencia adoptan una metodología de trabajo por proyectos.

Agradecimientos

La autora expresa su agradecimiento por la beca concedida por el Ministerio de Educación, Cultura y Deporte (España) en el programa "Formación del Profesorado Universitario".

\section{REFERENCIAS}

Ardanuy Baró, J. 2013. "La investigación científica catalana en Biblioteconomía y Documentación vista a través del Web of Knowledge”. Revista Española de Documentación Cientifica 36 (3): 1-7.

Arquero Avilés, R. y J. A. Salvador Oliván. 2004. "Evaluación y análisis de la producción de los autores de trabajos difundidos en publicaciones periódicas editadas en español: área de Biblioteconomía y Documentación: 1975-1984”. Documentación de las Ciencias de la Información 27: 53-74.

Bergillos, I. y D. Fernández Quijada. 2012. "Producción científica en comunicación en las universidades de la Comunidad de Madrid: la fuerza de la tradición”. Documentación de las Ciencias de la Información 35: 155-179.

Carpenter, J. 2011. Project management in libraries, archives and museums: working with government and other external partners. Oxford: Chandos.

Corral, S. 2000. Strategic management of information services: a planning handbook. London; ASILB. 
Eíto, R. 2006. “Lisa vs Lista: estudio Comparativo”. El Profesional de la Información 15 (6): 436-450.

Gómez Merino, R. y R. D. Santaella Ruiz. 2012. "Análisis de la producción científica sobre estudios de usuarios en la base de datos del CSIC de Biblioteconomía y Documentación”. Boletín de la Asociación Andaluza de Bibliotecarios 27 (104): 8-25.

Herrero Solana, V. y G. Liberatore. 2008. "Visibilidad internacional de las revistas iberoamericanas de Bibliotecología y Documentación”. Revista Española de Documentación Científica 31 (2): 230-239.

Herrero-Solana, V. y C. Ríos-Gómez. 2006. "Producción latinoamericana en Biblioteconomía y Documentación en el Social Science Citation Index (SSCI) 19662003". Information Research 11 (2).

Jiménez Contreras, E. 2002. "La aportación española a la producción científica internacional en Biblioteconomía y Documentación: balance de diez años (19922001)”. BiD: Textos Universitaris de Biblioteconomia i Documentació 9.

Jiménez Contreras, E., E. Delgado López-Cózar y R. Ruiz Pérez. 2006. "Producción española en Biblioteconomía y Documentación con visibilidad internacional a través del Web of Science (1995-2004)”. El Profesional de la Información 15 (5): 373-383.

Jiménez Contreras, E. y F. Moya Anegón. 1997. "Análisis de la autoría en revistas españolas de Biblioteconomía y Documentación: 1975-1995”. Revista Española de Documentación Científica 20 (3): 252-266.

Liberatore, G. 2015. "Análisis bibliométrico de la producción científica en Bibliotecología y Ciencia de la Información en Brasil en el periodo 2000-2011: estudio de cuatro revistas nacionales de la disciplina”. Tesis doctoral, Madrid: Universidad Carlos III de Madrid, Departamento de Biblioteconomía y Documentación.

Licea de Arenas, J., J. Valles, G. Arévalo y C. Cervantes. 2000. “Una visión bibliométrica de la investigación en Bibliotecología y Ciencia de la Información de América Latina y el Caribe”. Revista Española de Documentación Científica 23 (1): 45-53.

MacLachlan, Liz. 1996. Making project management work for you. London: Library Association.

Moore, K. 1998. “Project management: can libraries benefit?” Bibliotheca Medica Canadiana 20 (2): 72-73.

Morena de Diago, B. 2013. "Análisis de la Investigación Cualitativa en el área de Biblioteconomía y Documentación: (1981-2010)”. Revista General de Información y Documentación 23 (1): 43-64.

Moya Anegón, F. y V. Herrero-Solana. 2002. "Visibilidad internacional de la producción científica iberoamericana en Biblioteconomía y Documentación (19912000)". Ciência da Informação 31 (3): 54-65.

Project Management Institute. 2008. Guía de los fundamentos para la dirección de proyectos: (Guía del PMBOK), 4a. ed. Pennsylvania; Project Management Institute.

Torres-Salinas, D., E. Delgado López-Cózar y E. Jiménez-Contreras. 2009. “Análisis de la producción de la Universidad de Navarra en revistas de Ciencias Sociales y Humanidades empleando rankings de revistas españolas y la Web of Science". Revista Española de Documentación Científica 32 (1): 22-39.

Winston, Mark D. y Tara Hoffman. 2005. "Project management in libraries". Journal of Library Administration 42 (1): 51-61. 
Para citar este texto:

Cobo Serrano, Silvia. 2018. "Producción científica internacional sobre gestión de proyectos en el área de Información y Documentación: 1996-2015”. Investigación Bibliotecológica: archivonomía, bibliotecología e información 32 (75): 125-144.

http://dx.doi.org/10.22201/iibi.24488321xe.2018.75.57959 